\title{
Abordagem restauradora direta em dentes escurecidos: revisão de literatura
}

\author{
Direct restorative approach in darkened teeth: literature review \\ Abordaje restaurativo directo en dientes oscurecidos: revisión de la literatura
}

Recebido: 10/11/2021 | Revisado: 15/11/2021 | Aceito: 20/11/2021 | Publicado: 02/12/2021

\author{
Jatyra Souza Barbosa \\ ORCID: https://orcid.org/0000-0001-5427-3483 \\ Faculdade Independente do Nordeste, Brasil \\ E-mail: jatyrasouza@gmail.com \\ Anna Luisa Azevedo Dias Neres \\ ORCID: https://orcid.org/0000-0002-1167-4532 \\ Faculdade Independente do Nordeste, Brasil \\ E-mail: annaluisadneres@gmail.com \\ Saryta Argolo Souza Amaral \\ ORCID: https://orcid.org/0000-0002-2210-9758 \\ Faculdade Independente do Nordeste, Brasil \\ E-mail:Saryta@fainor.com.br
}

\begin{abstract}
Resumo
Introdução: A procura por tratamentos dentários restauradores vêm crescendo ao longo dos últimos anos. As alterações de cor dos dentes anteriores acarretam maior impacto estético, devido a sua maior visibilidade no momento de sorrir e de se comunicar. Objetivo: revisar a literatura sobre a abordagem das técnicas restauradoras para realização de restaurações diretas em dentes escurecidos. Metodologia: foi realizada revisão bibiliográfica nas bases de dados Pubmed, Scielo, BBO e LILACS. Foram selecionados artigos, publicações e pesquisas publicadas no período de 2015 a 2021 que estivessem relacionados à temática desse estudo. Resultados: o grande desafio na confecção de facetas diretas em dentes escurecidos é o mascaramento do substrato alterado. Para minimizar esse efeito indesejado o profissional pode aumentar o desgaste dental, ou de forma mais conservadora, utilizar os opacificadores, definido como resinas capazes de impedir a passagem de luz e mascarar cores indesejadas do substrato dental. Conclusão: o correto diagnóstico do escurecimento dentário além do domínio na manipulação e o conhecimento adequado dos agentes opacificadores são essenciais para a obtenção de uma estética natural do sorriso e o sucesso no tratamento com facetas diretas de dentes anteriores. A utilização de resinas opacificadoras é coadjuvante na técnica de faceta direta em dentes escurecidos e parece trazer resultados satisfatórios.
\end{abstract}

Palavras-chave: Facetas diretas; Dentes anteriores; Resina composta.

\begin{abstract}
Introduction: The demand for restorative dental treatments has been growing over the last few years. Changes in the color of anterior teeth have a greater aesthetic impact, due to their greater visibility when smiling and communicating. Objective: to review the literature on the approach of restorative techniques for direct restorations in darkened teeth. Methodology: a bibliographic review was carried out in the Pubmed, Scielo, BBO and LILACS databases. Articles, publications and research published in the period from 2015 to 2021 that were related to the subject of this study were selected. Results: the great challenge in making direct veneers on darkened teeth is the masking of the altered substrate. To minimize this unwanted effect, the professional can increase dental wear, or in a more conservative way, use opacifiers, defined as resins capable of preventing the passage of light and masking unwanted colors from the dental substrate. Conclusion: the correct diagnosis of tooth darkening, in addition to mastering the manipulation and adequate knowledge of opacifying agents are essential to obtain a natural smile esthetics and success in the treatment with direct veneers of anterior teeth. The use of opacifying resins is an adjunct in the direct veneer technique for darkened teeth and seems to bring satisfactory results.
\end{abstract}

Keywords: Direct facets; Anterior teeth; Composite resin.

\section{Resumen}

Introducción: La demanda de tratamientos dentales restauradores ha ido creciendo en los últimos años. Los cambios en el color de los dientes anteriores tienen un mayor impacto estético, debido a su mayor visibilidad al sonreír y comunicarse. Objetivo: revisar la literatura sobre el abordaje de las técnicas restauradoras para restauraciones directas en dientes oscurecidos. Metodología: se realizó una revisión bibliográfica en las bases de datos Pubmed, Scielo, BBO y LILACS. Se seleccionaron artículos, publicaciones e investigaciones publicadas en el período de 2015 a 2021 que tuvieran relación con el tema de este estudio. Resultados: el gran desafío en la realización de carillas directas sobre dientes oscurecidos es el enmascaramiento del sustrato alterado. Para minimizar este efecto no deseado, el profesional puede incrementar el desgaste dental, o de forma más conservadora, utilizar opacificantes, definidos como resinas 
capaces de evitar el paso de la luz y enmascarar los colores no deseados del sustrato dental. Conclusión: el correcto diagnóstico del oscurecimiento dental, además del dominio de la manipulación y el conocimiento adecuado de los agentes opacificantes son fundamentales para obtener una estética de sonrisa natural y éxito en el tratamiento con carillas directas de dientes anteriores. El uso de resinas opacificantes es un complemento de la técnica de revestimiento directo para dientes oscurecidos y parece dar resultados satisfactorios.

Palabras clave: Facetas directas; Dientes anteriores; Resina compuesta.

\section{Introdução}

Na sociedade atual, a busca por um sorriso harmônico e saudável tem se tornado algo cada vez mais comum. Desta forma os profissionais estão cada vez mais atentos a essa crescente demanda e ao desenvolvimento de novas técnicas e materiais com finalidade estética no intuito de devolver a autoestima e garantir resultados satisfatórios aos anseios de seus pacientes. Entretanto, não se pode esquecer as limitações que cada procedimento oferece. Deve-se respeitar as indicações e contraindicações de cada caso, selecionando a melhor alternativa de tratamento e agindo dentro da segurança e da ética, no intuito de buscar o equilíbrio entre a física e a biologia, alcançando assim saúde bucal (Cobb \&Wilson \& Grusak, 2018).

O escurecimento dental é causa de insatisfação e sempre foi uma preocupação dos profissionais em buscar formas de melhorar essa condição (Cabral \& Trauth, 2017). Pode ser causada por uma variedade de fatores, incluindo o estado da vitalidade pulpar, uso de medicamentos, dieta, má formação dentária, fluorose, hemorragias pulpares, pigmentação por materiais obturadores entre outros.

A faceta direta em resina composta é uma intervenção restauradora bastante aceita e eficaz no tratamento de dentes com alteração de cor na região anterior. Neste procedimento, recobre-se a face vestibular do substrato dentário por um material restaurador unido ao dente por meio do sistema adesivo (Fahl, 2016). As restaurações diretas têm se destacado por ser uma técnica rápida, segura e eficaz; menor custo em relação às cerâmicas, dispensam etapas de laboratório e não requerem provisório, nem moldagem (Oliveira et al. 2019). A evolução das propriedades físicas das resinas compostas, aliadas às suas características de estética e preservação de tecido dentário sadio, consolidou o uso destes materiais (Fahl, 2016).

No entanto, o sucesso estético destes procedimentos restauradores enfrenta enormes desafios. O uso de pigmentos ou compósitos opacificadores se fazem bastante eficazes (Cabral \& Trauth, 2017; Reis et al. 2018) e o entendimento adequado desses agentes são de extrema importância para atingir um equilíbrio entre a profundidade do preparo e a opacidade dos materiais restauradores para o sucesso do tratamento diante do escurecimento dental (Oliveira et al. 2019).

Diante disto, o objetivo do presente estudo é revisar a literatura sobre a abordagem das técnicas restauradoras para realização das restaurações diretas em dentes escurecidos.

\section{Metodologia}

Trata-se de uma revisão de literatura ou pesquisa integrativa cujo o método de pesquisa está baseado na integração de dados obtidos em bibliografias ou pesquisas de uma determinada temática (Pereira et al. 2018). Foram realizadas buscas por literaturas cientificas, em bases de dados, utilizando operador booleano "OR" combinando os seguintes descritores: "Facetas Diretas" "Dentes Anteriores" "Resina Composta". As bases de dados utilizadas foram Pubmed, Scielo, BBO - Bibliografia Brasileira de Odontologia, LILACS, sendo incluídos artigos, estudos e pesquisas publicadas no período de 2015 a 2020 , fundamentando-se em publicações atualizadas da literatura especializada, abrangendo artigos em inglês, espanhol e português o que nos possibilitou um panorama abrangente no que se refere à evolução de tais técnicas. Foram excluídos artigos sem correlação com o tema e fora do período citado. Sendo assim, foram selecionados 21 artigos que embasaram o presente estudo.

A fim de definir claramente a adequação da literatura encontrada neste estudo de revisão, para esse estudo foram estabelecidos os seguintes critérios de inclusão: a) Estudos com intervenções, sugerindo conteúdos relacionados à faceta direta 
dos dentes anteriores, incluindo suas vantagens e limitações; b) Publicação entre 2015 e intervalo de 2021 anos. Além de excluir artigos que não atendam aos parâmetros listados acima, os critérios de exclusão a) Pesquisas sem formato de texto completo; b) Pesquisas repetitivas fornecidas em múltiplas bases de dados; c) Pesquisas que não correspondem às palavraschave; d) Monografias e) Pesquisas que não envolvem tópicos. Na primeira verificação realizada, com base no título e resumo do artigo, foram rejeitados aqueles que não atendiam aos critérios de inclusão ou possuíam algum critério de exclusão. Após, foi sintetizado o texto, organizado o artigo para facilitar a compreensão dos dados e o processo de análise.

\section{Revisão de Literatura}

A faceta em resina Composta vêm sendo cada vez mais realizada pelos dentistas em seus consultórios. Inicialmente, uma boa anamnese pode trazer informações sobre a história clínica do paciente, hábitos de higiene, dieta e existência de parafuncões. Tais informações podem contraindicar ou indicar o uso de resinas compostas. A posição do dente na arcada e a extensão do preparo cavitário também devem ser levadas em conta durante a escolha do melhor procedimento. De forma que a obtenção do sucesso clínico de um trabalho restaurador perpassa por uma criteriosa seleção do caso proposto, observando todos os fatores envolvidos, o que vai determinar com mais certeza se a abordagem restauradora direta é apropriada para o caso (Velo, et al. 2016).

Em se tratando de escurecimento dental, sabe-se que esse processo ocorre por envelhecimento dental natural ou por fatores denominados extrínsecos ou intrínsecos. As pigmentações intrínsecas (também ditas manchas internas) podem estar ligadas à fatores como genética, idade, alterações na formação dos dentes, doenças congênitas, traumatismos dentais, uso de antibióticos específicos. Algumas restaurações também podem causar manchas intrínsecas (Trevisan et al. 2018). As manchas extrínsecas são manchas externas causadas de forma direta ou indireta. Normalmente causadas diretamente por hábitos dietéticos associados ao consumo excessivo de café, chá, vinho tinto, beterraba, cigarro, bem como hábitos de higiene do paciente; e, indiretamente, causadas por medicamentos locais ou antissépticos bucais como a clorexidina e cloreto cetilpiridínio (Martins \& Botelho \& Klug, 2021).

De fato, o comprometimento estético resultante de manchamentos dentários, causado na maioria das vezes por patologias e/ou efeitos colaterais de tratamentos, interferem fortemente de forma negativa na autoestima, sociabilidade, comportamento e relações interpessoais do individuo (Rezende \& Fajardo, 2016).

A execução das facetas diretas tem a missão de harmonizar e mimetizar os dentes através de compósitos, de forma minimamente invasiva. No planejamento restaurador sugere-se a opacificação das unidades escurecidas com corantes ou resinas compostas opacas de alto valor para possibilitar a homogeneidade nas cores dos substratos. É de importância o conhecimento da relação translucidez / opacidade de diversos sistemas de resinas compostas, auxiliando o mesmo na escolha do sistema ideal para cada caso (Mont', et al. 2019). Em relação aos compósitos, os profissionais têm uma infinidade de características cromáticas e indicações disponíveis no mercado, com o objetivo de replicar os dentes naturais, porém, toda essa variedade de opções por vezes aumentam as possibilidades e consequentemente a margem de erro, no entanto, quando o tratamento é realizado de forma planejada e minunciosamente seguindo o passo a passo, o resultado final tende a surpreender de forma positiva, devolvendo aos dentes o aspecto de dentes naturais (Gregorini, 2018).

O desenvolvimento da Odontologia adesiva proporcionou a melhora da união biomecânica entre dente e restauração, otimizando os procedimentos restauradores e permitindo uma abordagem mais conservadora e menos invasiva (Bispo, 2018; Goyatá et al., 2017). As facetas podem ser de dois tipos: diretas ou indiretas. As realizadas pelo próprio cirurgião-dentista são as diretas, que podem ser confeccionadas a partir de resinas compostas. As indiretas envolvem ou não o serviço laboratorial do técnico em prótese e podem constituir-se de resinas compostas ou de cerâmica (Soares et al. 2016). As restaurações diretas 
com resina composta resultam em procedimentos com uma maior preservação da estrutura dental em comparação com as restaurações indiretas (Menezes, et al. 2015).

$\mathrm{Na}$ odontologia atual se preserva o máximo de estrutura dentária e quando se trata de um dente escurecido a forma do preparo e o uso de opacificadores para mascarar aquele substrato é de extrema importância. No estudo de Felippe, et al. (2016) mostram que existem diferentes tonalidades de opacificadores. Terry (2015) em seu caso clínico, afirma que a depender do preparo realizado não se faz necessário o uso desse material opaco, porém, se faz relevante um preparo mais profundo ou criar um sobrecontorno intencional para o alinhamento do dente.

Em um relato de caso clínico apresentado por Okuda (2017), mostra que para realizar um preparo dental deve-se levar em consideração o limite gengival, proximal, incisal e profundidade do preparo. Neste estudo, quando foram retirados $0,7 \mathrm{~mm}$ das faces vestibular, incisal e proximal foi possível visualizar bem a cor interna do elemento dentário. Já Felippe, et al. (2016) dizem que em dentes manchados pelo uso da tetracilina por possuir uma dentina intensa e hipercromada, os autores sugerem $0,3 \mathrm{~mm}$ adicionais de desgastes totalizando pelo menos $1 \mathrm{~mm}$ de distância das margens.

Antes de realizar qualquer procedimento restaurador é necessário realizar um enceramento diagnóstico. No caso proposto por Terry (2015), um modelo em gesso permite ao paciente visualizar o tratamento e também serve como guia para definir a forma do dente. No caso proposto por D'Altoé (2015), foi confeccionado o enceramento e duas guias em silicone de condensação (SiIon 2 APS, Dentsply, Brasil), com cortes no sentido mesio-distal e o outro foi cortado na vestibular, terço incisivo, médio e cervical. Os autores realizaram ensaios restauradores na qual foi possível verificar a altura das restaurações além de reproduzir com detalhes a posição correta da borda incisal dos dentes que estavam sendo realizado o tratamento.

O modelo de estudo citado por Terry em 2015 trata-se do "Mockup" que é uma técnica interessante principalmente em dentes escurecidos pois deve-se visualizar a extensão cervical que deve ser ligeiramente subgengival com o objetivo de esconder a interface dente/restauração, bem como, mascarar qualquer diferença de cor que possa existir entre dente e material restaurador nessa região. Deve-se levar em consideração na decisão de estender o término cervical do preparo, a altura do sorriso do paciente (D'atloé, 2015).

Na confecção de faceta direta deve-se obter ponto de contato adequado e uma excelente adaptação marginal, livre de espaços e porosidades, portanto, após o término das restaurações devem-se avaliar os contatos oclusais em máxima intercuspidação habitual, em movimentos excursivos, de lateralidade e protrusão, garantindo assim, a análise oclusal estática e dinâmica preservando a longevidade e o sucesso do tratamento (Bispo, 2018).

O processo de acabamento e polimento pode afetar muitos aspectos da restauração final, incluindo a coloração da superfície, o acúmulo de placa bacteriana e as características de desgaste da resina. Portanto, são procedimentos primordiais para prolongar a longevidade do procedimento, proporcionando um excelente resultado estético final, através da execução de um protocolo baseado, em princípios de estética e harmonia dental (Costa \& Novais \& Carlos, 2020).

\section{Resultados}

O presente trabalho avaliou por meio de uma revisão de literatura os aspectos importantes sobre as facetas diretas em resina composta em dentes escurecidos utilizando-se de estratégias de pesquisa descrita que utilizou as palavras-chave: "facetas diretas", "dentes anteriores", e "resina composta". A princípio, foram realizadas coletas dos dados contendo as informações dos artigos a respeito das facetas em resina composta, por meio do levantamento bibliográfico que estarão apresentadas na Tabela 1 . 
Tabela 1. Resultados do levantamento bibliográfico.

\begin{tabular}{|c|c|c|}
\hline AUTOR/ANO & TÍTULO & RESULTADOS \\
\hline $\begin{array}{l}\text { SPAVERAS et al. } \\
\quad 2015\end{array}$ & $\begin{array}{l}\text { Masking the Discolored Enamel } \\
\text { Surface with Opaquers before Direct } \\
\text { Composite Veneering Spave. }\end{array}$ & $\begin{array}{l}\text { Os resultados apresentam que pacientes que apresentam bruxismo ficam } \\
\text { impossibilitado de utilizar facetas em resina composta. }\end{array}$ \\
\hline $\begin{array}{l}\text { FELIPPE } \text { et al., } \\
2016 .\end{array}$ & $\begin{array}{l}\text { Using opaquers under direct composite } \\
\text { resin veneers: an illustrated review of } \\
\text { the technique }\end{array}$ & $\begin{array}{l}\text { Os resultados mostram que a longevidade das facetas diretas em resina } \\
\text { compostas está relacionada a visita periódica ao dentista e com a higiene } \\
\text { do paciente e dos hábitos deletérios }\end{array}$ \\
\hline OKUDA, 2017. & $\begin{array}{l}\text { Using a modified subopaquing } \\
\text { technique to treat highly discolored } \\
\text { dentition }\end{array}$ & $\begin{array}{l}\text { Autor conclui que o uso do opacificador na técnica direta parece } \\
\text { desempenhar um papel interessante no mascaramento do fundo escuro, } \\
\text { porém, sob condições estéticas, não permite a passagem da luz, tornando a } \\
\text { restauração opaca e não translúcida. }\end{array}$ \\
\hline $\begin{array}{l}\text { FELIPPE \& } \\
\text { BARATIERI, } \\
2015 .\end{array}$ & $\begin{array}{l}\text { Direct resin composite veneers: } \\
\text { masking the dark prepared enamel } \\
\text { surface. }\end{array}$ & $\begin{array}{l}\text { Observa-se que para dente escurecido, um preparo ideal é de } 1 \text { a } 1,5 \mathrm{~mm} \text { de } \\
\text { desgaste, além da utilização do opacificador, caso contrário, o fundo escuro } \\
\text { dos dentes poderá ficar visível, e o resultado da cor será um tom de cinza. } \\
\text { Sendo assim, autores consideram um desgaste maior em dentes } \\
\text { escurecidos, mesmo utilizando uma resina opaca. }\end{array}$ \\
\hline $\begin{array}{l}\text { MENDONÇA \& } \\
\text { REINA, } 2018\end{array}$ & $\begin{array}{l}\text { Facetas esteticas directas de composite: } \\
\text { uso de opacificadores y tintes }\end{array}$ & $\begin{array}{l}\text { Enfatizam a importância da integração estética e integração funcional, } \\
\text { esses dois pontos estão relacionados ao perfeito entendimento da estrutura } \\
\text { anatômica natural dos dentes. }\end{array}$ \\
\hline $\begin{array}{l}\text { WEI \& TANG, } \\
2018\end{array}$ & $\begin{array}{l}\text { Laminate veneers for the aesthetic } \\
\text { restoration of anterior teeth. . }\end{array}$ & $\begin{array}{l}\text { Os resultados mostram que as pessoas podem questionar a eficácia do olho } \\
\text { humano em detectar as nuances dos dentes naturais neste processo, e então } \\
\text { será interessante comparar métodos visuais com espectrofotometria para } \\
\text { avaliar a integração óptica de restaurações anteriores. }\end{array}$ \\
\hline
\end{tabular}

Fonte: Autores (2021).

\section{Discussão}

É um grande desafio para o profissional mascarar cor em dente escurecido, e quando se encontra nessa situação clínica deve-se decidir sobre o tratamento mais adequado. Segundo Spaveras et al. (2015) alguns motivos devem ser levados em consideração. O bruxismo tem como exemplo de uma contra indicação da faceta e favorece o uso da cora total, outro motivo relevante é se há remanescente suficiente que consiga promover a adesão. Esse mesmo autor pontua em seu estudo clinico que dentes com escurecimento apresentam resultados insatisfatórios quando se utiliza a resina composta. Porém Felippe, et al. (2016) que essa técnica é extremamente viável e de grande excelência nesses tratamentos.

Felippe e Baratieri (2015) também observaram a espessura da resina composta é suficiente (cerca de $1 \mathrm{a} 1,5 \mathrm{~mm}$ ), com isso opacidade será insuficiente e o fundo escuro dos dentes será visível, e o resultado da cor será um tom de cinza. Apesar da utilização de resina hibridas opaca este problema não pode ser resolvido aplicando uma camada fina de resina sobre um preparo com grande pigmentação escura devido ao fato que mesmo camadas finas, opacas, têm grande potencial de opacidade. Segundo Spaveras et al. (2015) bons resultados podem ser obtidos por meio de preparo dentário conservador e adequado devido ao fato dos opacos apresentarem um ótimo potencial opacificador mesmo com finas camadas, eles permitem assim um bom efeito com um preparo dental conservador e adequado contorno da faceta.

Acredita-se que a técnica de faceta direta em resina composta é um procedimento restaurador estético, que possui várias vantagens, pois permite: realizar reparações no decorrer do procedimento, controle de cor e forma, eficazes e fáceis de confeccionar quando comparadas as faceta de cerâmica, que não necessitam de trabalho laboratorial, técnica minimamente invasiva, portanto mais conservadora, geralmente realizada em sessão única, resistência e estabilidade de cor favorável, dispensando a confecção de provisório, ótimo resultado estético, boa relação custo/benefício e menor tempo clínico, além de proporcionarem uma expectativa de longevidade clínica, em torno de 10 anos. Porém, requer que o profissional da Odontologia domine conhecimentos técnicos e científicos bastante específicos e que se utilize de cuidados especiais, sejam com as estruturas dentais ou com toda a área que recobre a gengival (Spaveras et al. 2015); (Okuda, 2017).

O sucesso desta tecnologia depende de dois pontos básicos: sua integração estética e sua integração funcional. Esses dois pontos estão relacionados ao perfeito entendimento da anatomia natural do dente e à dificuldade em encontrar o tom de 
resina composta correspondente para se obtiver uma restauração estética (Mendonça \& Reina, 2018). Portanto, as pessoas podem questionar a eficácia do olho humano em detectar as nuances dos dentes naturais neste processo, e então será interessante comparar métodos visuais com espectrofotometria para avaliar a integração óptica de restaurações dentárias anteriores (Wei \& Tang, 2018).

\section{Considerações Finais}

Diante do avanço da Odontologia adesiva e dos materiais restauradores diretos, a técnica de faceta direta em resina composta se tornou uma excelente opção de tratamento para reabilitação. Para obter um bom resultado é de extrema importância o planejamento, que dependerá da interação de conhecimentos sobre estética, adesão, materiais restauradores diretos e técnicas disponíveis atualmente. Para o tratamento em dentes com escurecimento dental, tem-se como aliado ao uso da resina composta, os materiais opacificadores, que por sua vez se tornam uma alternativa eficaz além de minimamente invasivo, atendendo as expectativas em relação ao mascaramento do substrato escuro.

\section{Referências}

Bispo, B. L. (2018). Laminados cerâmicos na clínica integrada. Revista de Odontologia da Universidade Cidade de São Paulo, 30(1),1-12. https://doi.org/10.26843/ro_unicidv3012018p83-94

Cabral, D. G. M., \& Trauth, K. G. S. (2017). Avaliação in vitro da técnica de mascaramento de substratos escuros com facetas de cerâmica através da estratificação sobre refratário. Revista de Odontologia da Universidade de Sao paulo, 22 (1), 1-9.

Cobb, A. B., Wilson, G., Goad, C. L., \& Grusak, M. A. (2018). Influence of alternative soil amendments on mycorrhizal fungi and cowpea production. Heliyon, 4(7), 1-7. https://dx.doi.org/10.1016\%2Fj.heliyon.2018.e00704

Costa, J. A., Novais, J. M., Carlos, A. M. P., \& Carlos, A. M. P. (2020). Use Of Opacifying Resins In Anterior Teeh With Intrinsic Staining-Literature Review. Braz. J. of , 9(7),1-9. 10.34117/bjdv6n11-657

D'Altoé, L. F. (2015) Faceta direta com resinas compostas para correção de forma, cor e posicionamento: relato de caso clinico. Clinica- International Journal of Brazilian Dentistry, 1(3), 1-8. 10.4034/revico.2019.17.2.12

Fahl Júnior N. (2016). The direct/indirect composite resin veneers: a case report. Practical periodontics and aesthetic dentistry: PPAD, 8(7),627-640.

Felippe, L. A., \& Baratieri, L. N. (2015). Direct resin composite veneers: masking the dark prepared enamel surface. Quintessence international. 31(8), 557562 .

Felippe, L. A., Monteiro, S., Jr, Baratieri, L. N., Caldeira de Andrada, M. A., \& Ritter, A. V. (2016). Using opaquers under direct composite resin veneers: an illustrated review of the technique. Journal of esthetic and restorative dentistry: official publication of the American Academy of Esthetic Dentistry, $15(6), 1-7$.

Martins, I. O., Botelho, S. S., \& Klug R. J. (2021). Solução Estética: Mascaramento em Dente Escurecido. Facit Business and Technology Journal,1 (28),1-8. https://doi.org/10.21270/archi.v6i8.2218

Menezes1, M., Carvalho, E., Silva, F., Reis, G., \& Borges, M. (2015). Caso Clínico Reabilitação estética do sorriso com laminados cerâmicos: Relato de caso clínico Aesthetic Restabilishment with laminates venners, 23(68),1-7.

Mont', B., Haddade, A., Francisco, S., Ribeiro, C., Mont'alverne, G., Pires, M., Celso, S., Da, A., \& Rodrigues Filho, S. (2019). Soluções Estéticas Diretas Em Dentes Escurecidos -Relato de Caso. Direct Aesthetic Solutions On Darkened Teeth -Case Report, 30(1), 83-94 10.34117/bjdv6n11-657.

Okuda W. H. (2017). Using a modified subopaquing technique to treat highly discolored dentition. Journal of the American Dental Association, 131(7), 945950. 10.14219/jada.archive.2000.0313

Oliveira, S., Alexandre, E., Héllen de Lacerda Oliveira, Ventura, P., \& Sonia Luque Peralta. (2019). Mascaramento de dentes escurecidos utilizando restaurações diretas: relato de caso. Revista Diálogos Acadêmicos, 8(2).

Pereira,A. S.,Shitsuka, D. M., Parreira, F. J., \& Shitsuka, R. (2018). Metodologia da pesquisa científica. UFSM. https://repositorio.ufsm.br/bitstream/handle/1/15824/Lic_Computacao_Metodologia-Pesquisa-Cientifica.pdf?sequence=1.

Reis, G. R., Oliveira, L. P. M., Vilela, A. L. R., \& Menezes, M. D. S. (2018). Mock-up: previsibilidade e facilitador das restaurações estéticas em resina composta. Rev Odontol Bras Central, 27(81), 105-111

Soares, p. V., Reinke, A. C. M. A., Moura, G. F., Zeola, L. F., Machado, A. C., \& Reis, B. R. (2016). Reabilitação estética e funcional com facetas diretas após histórico de traumatismo dento-alveolar. Revista Odontologica Do Brasil Central, 25(74),1-8. Doi.org/10.36065/robrac.v25i74.1057 .

Spaveras A, Vjero O,Anagnostou M, \& Antoniadou M (2015). Masking the Discolored Enamel Surface with Opaquers before Direct Composite Veneering Spave. J Dent Oral Disord Ther, 3 (2), 1-8. 
Research, Society and Development, v. 10, n. 15, e500101523130, 2021

(CC BY 4.0) | ISSN 2525-3409 | DOI: http://dx.doi.org/10.33448/rsd-v10i15.23130

Terry D. A. (2015). Direct reconstruction of the maxillary anterior dentition with composite resin: a case report. Practical periodontics and aesthetic dentistr: PPAD, 11(3), 361-368.

Trevisan, T. C., Gusson Júnior, M., Bortolatto, J. F., Pigossi, S., Oliveira Júnior, O. B. D., \& Ricci, W. A. (2018). Color stability of conventional and bulk fill composite resins. RGO - Revista Gaúcha de Odontologia, 66(1), 15-20. https://doi.org/10.1590/1981-863720180001000023125

Velo, M. M. d. A. C., Coelho, L. V. B. F., Basting, R. T., Amaral, F. L. B. d., \& França, F. M. G. (2016). Longevity of restorations in direct composite resin: literature review. RGO - Revista Gaúcha de Odontologia, 64(3),320-326.https://doi.org/10.1590/1981-8637201600030000123109.

Wei, S. H., \& Tang, E. (2018). Laminate veneers for the aesthetic restoration of anterior teeth. Annals of the Royal Australasian College of Dental Surgeons, 10 (1) $148-159$. 